\title{
Bioanalysis
}

\section{A narrative for bioanalytical method development}

\author{
"Method development performed with a regulated bioanalysis \\ mindset may focus more on abiding to regulated bioanalysis \\ procedures and workflow rather than grasp the science at play."
}

First draft submitted: 13 November 2016; Accepted for publication: 30 November 2016; Published online: 13 January 2017

Keywords: breakthrough $\bullet$ exploratory $\bullet$ method development narrative $\bullet$ optimization - pre-validation $\bullet$ regulated bioanalysis $\bullet$ R\&D

Regulated bioanalysis (RB) oftentimes contradicts intrinsic $R \& D$ values. $R B$ is intended to comply with regulations and as such follows a fixed study plan and experimental procedure, whereas methods in development evolve dynamically. Method development (MD) performed with a RB mindset may focus more on abiding to RB procedures and workflow rather than grasp the science at play. Except for exploratory method validations (MV) for biomarkers [1] and such, it is typically not possible to 'fix' a failing validated method without revalidation, which further emphasizes the importance of adequate MD before moving on to MV.

If it is true that $\mathrm{R} \& \mathrm{D}$ can be difficult to predict in terms of outcomes, timelines and budget, bearing in mind a robust method for productive sample analyses is the objective, the bioanalytical MD cycle can be schematized as a narrative informally followed to achieve that very objective.

A tentative to circumscribe bioanalytical MD is set forth (Figure 1) that outlines three MD phases: the exploratory phase (EP), the optimization phase (OP) and the pre-validation phase (PV). In addition, the turning point in $\mathrm{MD}$ is described as the breakthrough (BT).

The EP is ground-breaking and uncertain by nature. The EP could also be called a feasibility phase because usually it is where the method will make or break. At onset, the EP seeks signal responses over noise to shape a standard curve. The EP then lets the experimenter test and qualifies the method parameters for assay functionality, inner workings and pitfalls. One can alternatively view the $\mathrm{EP}$ as a sequence of hypotheses testing or troubleshooting experiments. It is also a selfeducational crash course on the method. The EP can take the form of a method transfer. Nevertheless, the EP method is evolutive by nature and multiple iterations of the procedure are implemented with each assay leading to a functional assay.

Experience demonstrates that typically one major roadblock to assay functionality will be faced within or toward the end of the EP. The BT is defined as a solution to the key challenge with the method undergoing development. The BT is the turning point in the MD narrative because upon achieving the BT, likelihood for method feasibility increases drastically. It is therefore paramount to identify the primary roadblock during EP. The EP should define, describe, document the challenge, thereby suggesting hypotheses and solutions. Failure to appropriately identify and understand key method roadblocks along the way will result in lengthy $\mathrm{EP}$, in other words, endless circumvolutions around the problem. In such cases, the number of assays performed can rapidly explode. Examples of challenges include weak S/N ratio, inefficient or unspecific extraction, irreproducible results, insufficient sensitivity, lack of parallelism, unspecific signal, etc.

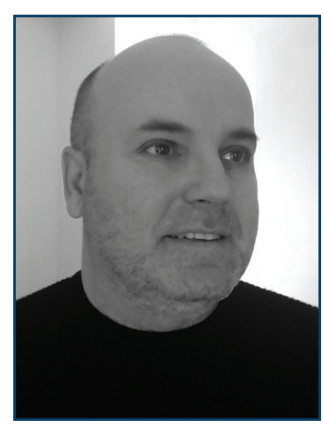

Guy Tremblay Oligenix, Montreal, QC, Canada Tel.: +1 5147096458 guy.tremblay@gmail.com 


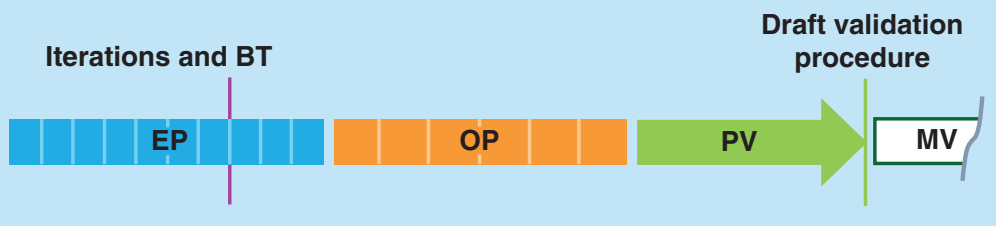

Figure 1. Schematic method development narrative. Vertical lines indicate iterations of and changes to the assay procedure. The magenta line corresponds to the method BT.

BT: Breakthrough; EP: Exploratory phase; MV: Method validations; OP: Optimization phase; PV: Pre-validation phase.

Basically, challenges vary wildly and each assay is different. Finally, achieving BT will roll the method on to the OP.

The OP fine-tunes the main method parameters. It can be described as reaching the $\mathrm{S} / \mathrm{N}$ maximum for each comprehensive variable. Examples include antibody titers, mobile-phase ratios, competitor concentration, minimal required dilution, incubation times and temperature, column temperature, extraction volume, etc. OP is also iterative in nature and ideally optimization is carried-out one variable at a time. With maxima obtained looking at relevant metrics, whether it concerns $\mathrm{S} / \mathrm{N}$ ratio at the lower end of the curve, curve shape, precision and accuracy, selectivity or else, the experimenter can move on to PV.

Last, but not least, the PV phase primes the upcoming regulation compliant MV. Oftentimes, assays performed in the EP will be repeated in a more rigorous way with the now optimized method in PV, for example, if selectivity was performed at the EP with only four lots of biological matrix, it will now be performed with ten lots as with the upcoming MV. The extent of the PV varies, sometimes only one assay is performed, depending on the perceived robustness of the method, since these parameters will be assessed in the formal MV. However, a thorough PV, with occasional return to EP or OP, should make the subsequent MV leaner and dependable. If the method is not intended to be validated, then the PV amounts to a qualification of the method and may be more extensive. Finally, PV concludes with draft-

\section{References}

1 Khan MN, Findlay JW. Ligand-Binding Assays: Development, Validation, and Implementation in the Drug Development Arena. Fit-For-Purpose Method Development and Validation. John Wiley \& Sons, New York, USA, 132-134 (2009).

2 Schmauser B. Analytical method development. Presented at: WHO/FIP Training Workshop on Pharmaceutical ing method procedure in preparation for MV. Drafting of the validation method by the development scientist transmits the science involved to RB.

Finally, it may be noteworthy to add that every method is different and as such the narrative is a general template for MD structure. For instance, roadblocks may be encountered during OP or PV. Also, there is a degree of crossover since, for instance, some optimization is inherently run concomitant to EP, and prevalidation parameters will be tested during EP and OP. Nevertheless, this simple MD narrative contributes to ground bioanalytical MD bearing in mind R\&D values and process in the context of RB. Realizing the narrative and identifying the key method challenges early on that lead to BT achievement will accelerate fit-for-purpose MD and help avoid method failure during RB. Furthermore, the bioanalytical MD narrative can broadly apply to various bioanalytical platforms as well as other fields that require regulated MV such as analytical MD [2] and microbiological MD [3].

\section{Financial \& competing interests disclosure}

The author has no relevant affiliations or financial involvement with any organization or entity with a financial interest in or financial conflict with the subject matter or materials discussed in the manuscript. This includes employment, consultancies, honoraria, stock ownership or options, expert testimony, grants or patents received or pending, or royalties.

No writing assistance was utilized in the production of this manuscript.

Development, with Focus on Paediatric Formulations. Beijing, PR of China, 21-24 June 2010.

3 Curren RD, Southee JA, Spielmann H, Liebsch M, Fentem JH, Balls M. The role of prevalidation in the development, validation and acceptance of alternative methods. ATLA 23, 211-217 (1995). 